Honam Mathematical J. 34 (2012), No. 3, pp. 439-449

http://dx.doi.org/10.5831/HMJ.2012.34.3.439

\title{
FIXED POINTS AND ALTERNATIVE PRINCIPLES
}

\author{
Sehie Park And Hoonjoo Kim
}

\begin{abstract}
In a recent paper, M. Balaj [B] established an alternative principle. The principle was applied to a matching theorem of Ky Fan type, an analytic alternative, a minimax inequality, and existence of solutions of a vector equilibrium theorem. Based on the first author's fixed point theorems, in the present paper, we obtain generalizations of the main result of Balaj [B] and their applications.
\end{abstract}

\section{Introduction}

In a recent paper, M. Balaj [B] established an alternative principle of the following type: If $X$ and $Y$ are convex subsets of two locally convex Hausdorff topological vector spaces and $F, S: X \multimap Y$ are two multimaps satisfying certain conditions, then either $F\left(x_{0}\right)=\emptyset$ for some $x_{0} \in X$ or $\bigcap_{x \in X} S(x) \neq \emptyset$. This principle was applied to a matching theorem of Ky Fan type, an analytic alternative, a minimax inequality, and existence of solutions of a vector equilibrium theorem.

Actually, Balaj based on a fixed point theorem of Lassonde [L] for compositions of Kakutani maps. Quite long time ago, the first author obtained several fixed point theorems [P1-7] generalizing many previously known ones including Lassonde's.

Based on the first author's fixed point theorems, in the present paper, we obtain generalizations of the main result of Balaj $[B]$ and their applications.

Received August 6, 2012. Accepted August 26, 2012.

2010 Mathematics Subject Classification. 47H10; 49J35; 52A07; 54H25; 54C60; $90 \mathrm{C} 47$.

Key words and phrases. Acyclic map; Fixed point; Matching theorem; Analytic alternative; Minimax inequality. 


\section{Known fixed point theorems}

Multimaps are called simply maps sometimes. For a multimap $T$ : $X \multimap Y$, three maps $T^{c}: X \multimap Y$ the complement of $T, T^{-}: Y \multimap X$ the (lower) inverse of $T$ and $T^{*}: Y \multimap X$, the dual of $T$ are defined by $T^{c}(x)=Y \backslash T(x), T^{-}(y)=\{x \in X \mid y \in T(x)\}$ and $T^{*}(y):=X \backslash T^{-}(y)$ resp.

For topological spaces $X$ and $Y$, a map $T: X \multimap Y$ is said to be: upper semicontinuous (u.s.c.) if for any closed set $B \subset Y$, the set $T^{-}(B)=\{x \in X \mid T(x) \cap B \neq \emptyset\}$ is closed in $X$; compact if $T(X)$ is contained in a compact subset of $Y$; closed if its graph is a closed subset of $X \times Y$.

The following is well-known:

Lemma 1. (i) A composition of u.s.c. maps with comapct values is u.s.c. with comapct values.

(ii) If $Y$ is compact and $T: X \multimap Y$ is closed, then $T$ is u.s.c. with compact values.

(iii) If $T$ is u.s.c. with compact values, then $T(K)$ is compact whenever $K \subset X$ is compact.

A map $T: X \multimap Y$ is called a Kakutani map whenever $X$ is a topological space, $Y$ a convex space (in the sense of Lassonde) and $T$ is u.s.c. with nonempty compact convex values. Let $\mathbb{K}(X, Y)$ be the class of all Kakutani maps $T: X \multimap Y$ and $\mathbb{K}_{c}(X, Y)$ the class of all maps $T: X \multimap Y$ those are finite compositions of Kakutani maps.

A topological space is said to be acyclic if all of its reduced Cech homology groups over the rational field vanish. A u.s.c. map is said to be acyclic if it has compact acyclic values. Let $\mathbb{V}(X, Y)$ be the class of all acyclic maps $T: X \multimap Y$ and $\mathbb{V}_{c}(X, Y)$ the class of all maps $T: X \multimap Y$ those are finite compositions of acyclic maps.

A polytope $P$ in a t.v.s. $E$ is a homeomorphic image of a simplex $\Delta_{n}$.

For topological spaces $X$ and $Y$, we define the "better" admissible class $\mathfrak{B}$ of maps from $X$ into $Y$ as follows [P3,4,7]:

$F \in \mathfrak{B}(X, Y) \Longleftrightarrow F: X \multimap Y$ is a map such that, for any natural number $n \in \mathbb{N}$, any continuous function $\phi: \Delta_{n} \rightarrow X$, and any continuous function $p: F \phi\left(\Delta_{n}\right) \rightarrow \Delta_{n}$, the composition

$$
\Delta_{n} \stackrel{\phi}{\longrightarrow} \phi\left(\Delta_{n}\right) \subset X \stackrel{F}{\longrightarrow} F \phi\left(\Delta_{n}\right) \stackrel{p}{\longrightarrow} \Delta_{n}
$$

has a fixed point. 
Examples. Subclasses of the better admissible class $\mathfrak{B}$ are classes of continuous functions $\mathbb{C}$, the Kakutani maps $\mathbb{K}$ (with convex values and codomains are convex spaces), the Aronszajn maps $\mathbb{M}$ (with $R_{\delta}$ values), the acyclic maps $\mathbb{V}$ (with acyclic values), the Powers maps $\mathbb{V}_{c}$ (finite compositions of acyclic maps), the O'Neill maps $\mathbb{N}$ (continuous with values of one or $m$ acyclic components, where $m$ is fixed), the approachable maps $\mathbb{A}$ (whose domains and codomains are subsets of t.v.s.), admissible maps of Górniewicz (1976), $\sigma$-selectionable maps of Haddad and Lasry (1983), permissible maps of Dzedzej (1985), the Fan-Browder maps (codomains are convex sets), locally selectionable maps having convex values, the class $\mathbb{K}_{c}^{+}$of Lassonde (1991), the class $\mathbb{V}_{c}^{+}$of Park et al. (1994), and approximable maps of Ben-El-Mechaiekh and Idzik (1994), the admissible class $\mathfrak{A}_{c}^{\kappa}$ due to Park (1993). For the literature, see [P1-4].

Let $X$ be a convex subset of a vector space and $Y$ a nonempty set. If $S, T: X \multimap Y$ are two maps such that $T(\operatorname{co} A) \subset S(A)$ for each nonempty finite subset $A$ of $X$, then $S$ is said to be $K K M$ w.r.t. T.

From now on, a t.v.s. means a Hausdorff topological vector space and a real locally convex Hausdorff topological vector space is abbreviated as an l.c.s.

Let $\mathcal{V}$ be the neighborhood system of the origin 0 of a t.v.s. $E$.

Recall that a nonempty subset $X$ of a t.v.s. $E$ is said to be admissible (in the sense of Klee) provided that, for every nonempty compact subset $K$ of $X$ and every $V \in \mathcal{V}$, there exists a continuous function $h: K \rightarrow X$ such that $x-h(x) \in V$ for all $x \in K$ and $h(K)$ is contained in a finite dimensional subspace $L$ of $E$.

A compact subset $K$ of $X$ is said to be Klee approximable into $X$ if for any $V \in \mathcal{V}$, there exists a continuous function $h: K \rightarrow X$ such that $x-h(x) \in V$ for all $x \in K$ and $h(K)$ is contained in a polytope in $X$.

Recall that a nonempty subset $Y$ of a t.v.s. $E$ is said to be almost convex if for any neighborhood $V$ of the origin 0 of $E$ and for any finite set $\left\{y_{1}, \ldots, y_{n}\right\}$ of $Y$, there exists a finite set $\left\{z_{1}, \ldots, z_{n}\right\}$ of $Y$ such that $z_{i}-y_{i} \in V$ for all $i=1, \ldots, n$ and $\operatorname{co}\left\{z_{1}, \ldots, z_{n}\right\} \subset Y$.

Examples. We give examples of Klee approximable sets in $[\mathrm{P} 5,6]$ :

(1) If a subset $X$ of $E$ is admissible (in the sense of Klee), then every compact subset $K$ of $X$ is Klee approximable into $E$.

(2) Any polytope in a subset $X$ of a t.v.s. is Klee approximable into $X$. 
(3) Any compact subset $K$ of a convex subset $X$ in an l.c.s. is Klee approximable into $X$.

(4) Any compact subset $K$ of a convex and locally convex subset $X$ of a t.v.s. is Klee approximable into $X$.

(5) Any compact subset $K$ of an admissible convex subset $X$ of a t.v.s. is Klee approximable into $X$.

(6) Let $X$ be an almost convex dense subset of an admissible subset $Y$ of a t.v.s. $E$. Then every compact subset $K$ of $Y$ is Klee approximable into $X$

Note that $(6) \Leftarrow(5) \Leftarrow(4) \Leftarrow(3)$.

In 2004 [P5], the first author obtained the following fixed point theorem:

Theorem 1. Let $X$ be a subset of a t.v.s. $E$ and $F \in \mathfrak{B}(X, X)$ a compact closed multimap. If $F(X)$ is Klee approximable into $X$, then $F$ has a fixed point.

The following are recently obtained in 2007 [P6]:

Corollary 1.1. Let $X$ be an almost convex admissible subset of a t.v.s. $E$ and $F \in \mathfrak{B}(X, X)$ a compact closed map. Then $F$ has a fixed point.

Corollary 1.2. Let $X$ be an almost convex subset of an l.c.s. $E$ and $F \in \mathfrak{B}(X, X)$ a compact closed map. Then $F$ has a fixed point.

One of the simplest known examples of Corollary 1.2 is that every compact continuous selfmap on an almost convex subset of a Euclidean space has a fixed point. This generalizes the Brouwer fixed point theorem.

Corollary 1.3. [P4] Let $X$ be an admissible convex subset of a t.v.s. E and $F \in \mathfrak{B}(X, X)$ a compact closed map. Then $F$ has a fixed point.

\section{The Balaj type alternative principles}

In this section, we apply our fixed point theorems to several alternative principles.

Theorem 2. Let $X$ be an almost convex admissible subset of a t.v.s. and $Y$ a topological space, one of them compact. Let $F, S: X \multimap Y$ be two maps satisfying the following conditions:

(1) for each $x \in X, F(x) \subset S(x)$;

(2) $F$ and $S^{*}$ are empty-valued or acyclic maps; 
Then at least one of the following assertions holds:

(a) There exists $x_{0} \in X$ such that $F\left(x_{0}\right)=\emptyset$.

(b) $\bigcap_{x \in X} S(x) \neq \emptyset$.

Proof. Suppose that assertions (a) and (b) are both false. Note that $\bigcap_{x \in X} S(x)=\emptyset$ is equivalent to the fact that the map $S^{*}$ has nonempty values. Hence both $F$ and $S^{*}$ have nonempty values. Hence, by (2), $F \in \mathbb{V}(X, Y)$ and $S^{*} \in \mathbb{V}(Y, X)$. Let us consider the map $H=S^{*} \circ F \in$ $\mathbb{V}_{c}(X, X) . \quad H$ is a u.s.c. map with compact values, so $H$ is closed. Since $\mathbb{V}_{c} \subset \mathfrak{B}$, Corollary 1.1 is applicable as soon as we prove that the map $H$ is compact. Clearly this happens if $X$ is compact. When $Y$ is compact, since $S^{*}$ is a u.s.c. map with compact values, $S^{*}(Y)$ is compact by Lemma 1(iii). Since $H(X) \subset S^{*}(Y), H$ is a compact map. By Corollary 1.1, $H$ has a fixed point. This implies that there exist $x_{0} \in X$ and $y_{0} \in Y$ such that $y_{0} \in F\left(x_{0}\right) \subset S\left(x_{0}\right)$ and $x_{0} \in S^{*}\left(y_{0}\right) ;$ a contradiction.

The following are variants of Theorem 2 :

Theorem 3. Let $X$ be an admissible convex subset of a t.v.s. and $Y$ a convex space, one of them compact. Let $F, S: X \multimap Y$ be two maps satisfying the following conditions:

(1) for each $x \in X, F(x) \subset S(x)$;

(2) $F$ and $S^{*}$ are empty-valued or Kakutani maps;

Then at least one of the following assertions holds:

(a) There exists $x_{0} \in X$ such that $F\left(x_{0}\right)=\emptyset$.

(b) $\bigcap_{x \in X} S(x) \neq \emptyset$.

Proof. As in the proof of Theorem 2, we can define $H=S^{*} \circ F \in$ $\mathbb{K}_{c}(X, X)$, where the intermediate space $Y$ is a convex space in the sense of Lassonde. Then we can apply Corollary 1.3.

Lemma 2. [B] Let $X$ be a topological space and $Y$ a nonempty convex set in an l.c.s. E. Let $T: X \multimap Y$ be a u.s.c. map with nonempty values such that $\overline{\operatorname{co}} T(x)$ is compact for each $x \in X$. Then the map $\overline{\operatorname{co}} T$ is u.s.c.

Theorem 4. Let $X$ be an almost convex admissible subset of a t.v.s. and $Y$ a nonempty convex subset in an l.c.s., one of them compact. Let $F, G, S: X \multimap Y$ be three maps satisfying the following conditions:

(1) for each $x \in X, \operatorname{co} F(x) \subset G(x) \subset S(x)$;

(2) $F$ is empty-valued or a u.s.c. map;

(3) $G$ has compact values;

(4) $S^{*}$ is empty-valued or an acyclic map; 
Then at least one of the following assertions holds:

(a) There exists $x_{0} \in X$ such that $F\left(x_{0}\right)=\emptyset$.

(b) $\bigcap_{x \in X} S(x) \neq \emptyset$.

Proof. Suppose that assertions (a) and (b) are both false. Then $F$ and $S^{*}$ have both nonempty values. By (1) and (3), $\overline{\operatorname{co}} F(x) \subset G(x) \subset Y$ and $\overline{\mathrm{co}} F(x)$ is a nonempty compact set, for each $x \in X$. By Lemma 2 , the map $\overline{\mathrm{co}} F$ is u.s.c., hence $\overline{\mathrm{co}} F \in \mathbb{K}(X, Y)$. As in the proof of Theorem 2, we can define $H=S^{*} \circ \overline{\mathrm{co}} F \in \mathbb{V}_{c}(X, X)$. Then we can apply Corollary 1.1 .

From now on, we consider the Balaj type alternative principles:

Theorem 5. Let $X$ be an admissible convex subset of a t.v.s. and $Y$ a nonempty convex subset in an l.c.s., one of them compact. Let $F, G, S, T: X \multimap Y$ be four maps satisfying the following conditions:

(1) for each $x \in X$, co $F(x) \subset G(x) \subset T(x)$;

(2) for each $y \in Y, \operatorname{co} S^{*}(y) \subset T^{*}(y)$;

(3) $F$ and $S^{*}$ are u.s.c.;

(4) $G$ and $T^{*}$ have compact values.

Then at least one of the following assertions holds:

(a) There exists $x_{0} \in X$ such that $F\left(x_{0}\right)=\emptyset$.

(b) $\bigcap_{x \in X} S(x) \neq \emptyset$.

Proof. Suppose that assertions (a) and (b) are false. Then $F$ and $S^{*}$ have nonempty values. By (1), (4) and Lemma 2, $\overline{\operatorname{co}} F \in \mathbb{K}(X, Y)$. Moreover, by (2) and (4), $\overline{\mathrm{co}} S^{*}(y) \subset T^{*}(y)$ so $\overline{\mathrm{co}} S^{*}(y)$ is compact for each $y \in Y$. By Lemma 2 , the map $\overline{\mathrm{co}} S^{*}$ is u.s.c., hence $\overline{\mathrm{co}} S^{*} \in \mathbb{K}(Y, X)$. Let us consider the map $H=\overline{\mathrm{co}} S^{*} \circ \overline{\mathrm{co}} F \in \mathbb{K}_{c}(X, X)$. Since $K_{c} \subset \mathfrak{B}$, and $H$ is closed and compact, $H$ has a fixed point by Corollary 1.3. This implies that there exist $x_{0} \in X$ and $y_{0} \in Y$ such that

$$
y_{0} \in \overline{\mathrm{co}} F\left(x_{0}\right) \subset G\left(x_{0}\right) \subset T\left(x_{0}\right) \text { and } x_{0} \in \overline{\mathrm{co}} S^{*}\left(y_{0}\right) \subset T^{*}\left(y_{0}\right) ;
$$

which is a contradiction.

Corollary 5.1. [B, Theorem 3.1] Let $X$ and $Y$ be nonempty convex sets in an l.c.s., and let $X$ or $Y$ be compact. Let $F, G, S, T: X \multimap Y$ be four maps satisfying the following conditions:

(i) for each $x \in X$, $\operatorname{co} F(x) \subset G(x) \subset T(x)$;

(ii) $S$ is a KKM map w.r.t. T;

(iii) $F$ and $S^{*}$ are u.s.c.;

(iv) $G$ and $T^{*}$ have compact values.

Then at least one of the following assertions holds:

(a) There exists $x_{0} \in X$ such that $F\left(x_{0}\right)=\emptyset$. 
(b) $\bigcap_{x \in X} S(x) \neq \emptyset$.

Proof. Comparing the requirements of Theorem 5 and Corollary 5.1, it suffices to show that

(2) for each $y \in Y, \operatorname{co} S^{*}(y) \subset T^{*}(y)$.

In fact, let $x \in \operatorname{co} S^{*}(y)$. Then there exists a finite set $A \subset S^{*}(y)$ such that $x \in \operatorname{co} A$. From $A \subset S^{*}(y)$ it follows at once, $y \notin S(A)$ and taking into account (ii), $y \notin T(\operatorname{co} A)$. Particularly $y \notin T(x)$, that is, $x \in T^{*}(y)$. Thus, (2) holds. Then we have the conclusion.

As it is mentioned in [B], the compactness condition, for at least one of the sets $X$ and $Y$, is essential in Corollary 5.1. But the example in [B] showing this fact must be corrected as follows:

Let $X=Y=\mathbb{R}$ and $F, G, S, T: \mathbb{R} \multimap \mathbb{R}$ be defined by $F(x)=\{x+$ $1, x+2\}, G(x)=[x+1, x+2], T(x)=\mathbb{R} \backslash[x-1, x]$, and $S(x)=\mathbb{R} \backslash\{x\}$ for all $x \in \mathbb{R}$.

In [B], Balaj applied his Theorems 3.1 to the following:

(1) a matching theorem of Ky Fan type,

(2) an analytic alternative,

(3) a minimax inequality, and

(4) existence of solutions of a vector equilibrium theorem.

Since we obtained improved versions of [B, Theorem 3.1], the above applications also can be improved. From Theorem 4, we also obtain a matching theorem of Ky Fan type, an analytic alternative, a minimax inequality as follows.

\section{Applications}

The following is Ky Fan type matching theorem [F];

Theorem 6. Let $X$ be an almost convex admissible subset of a t.v.s. and $Y$ a nonempty convex subset in an l.c.s., one of them compact. Let $\left\{B_{i}: i \in I\right\}$ be a family of closed subsets of $X$ such that $\bigcup_{i \in I} B_{i}=X$ and $\left\{x_{i}: i \in I\right\}$ be a family (indexed also by $I$ ) of points of $X$ and the set $\left\{i \in I: x \notin B_{i}\right\}$ is finite for each $x \in X$. Let $F, G, S: X \multimap Y$ be three maps satisfying the following conditions:

(1) for each $x \in X, S(x) \subset G(x)$;

(2) $F$ is a u.s.c. map;

(3) $G^{c}$ has compact values;

(4) $S(X)=Y$; 
(5) $S^{-}$is an acyclic map;

Then there exists a nonempty subset $J$ of I such that

$$
\operatorname{co} F\left(\left\{x_{i}: i \in J\right\}\right) \cap G\left(\cap\left\{B_{i}: i \in J\right\}\right) \neq \emptyset .
$$

Proof. For each $x \in X$, let $I(x)=\left\{i \in I: x \in B_{i}\right\}$. Then $I(x) \neq \emptyset$ for each $x \in X$, since $\left\{B_{i}: i \in I\right\}$ covers $X$. Define $H: X \multimap X$ by

$$
H(x)=\left\{x_{i}: i \in I(x)\right\} \text { for each } x \in X .
$$

Then $H$ has nonempty values. For each $x \in X$, let $U(x)=X \backslash \bigcup\left\{B_{i}\right.$ : $i \notin I(x)\}$. Then $U(x)$ is an open neighborhood of $x$ and, if $z \in U(x)$, then $H(z) \subset H(x)$. This shows $H$ is u.s.c.. Define the maps $F_{1}, G_{1}, S_{1}$ : $X \multimap Y$ by

$$
F_{1}=F \circ H, G_{1}=G^{c}, S_{1}=S^{c} .
$$

Note that $S_{1}^{*}=S^{-}$. From the hypothesis it readily follows that the maps $F_{1}, G_{1}, S_{1}$ satisfy the conditions (2), (3), (4) in Theorem 4 and $G_{1}(x) \subset S_{1}(x)$ for each $x \in X$. Since $F_{1}$ has nonempty values and by (4), $\bigcap_{x \in X} S_{1}(x)=\emptyset$, the conclusion of Theorem 4 does not hold. Consequently for some $x_{0} \in X, \operatorname{co}_{1}\left(x_{0}\right) \not \subset G_{1}\left(x_{0}\right)$ or equivalently,

$$
\operatorname{co} F\left(\left\{x_{i}: i \in I\left(x_{0}\right)\right\}\right) \cap G\left(x_{0}\right) \neq \emptyset .
$$

Since $x_{0} \in \bigcap\left\{B_{i}: i \in I\left(x_{0}\right)\right\}$, putting $J=I\left(x_{0}\right)$ we obtain that

$$
\operatorname{co} F\left(\left\{x_{i}: i \in J\right\}\right) \cap G\left(\cap\left\{B_{i}: i \in J\right\}\right) \neq \emptyset .
$$

Let $X$ be a convex subset of a vector space, $Y$ a nonempty set and $f, g: X \times Y \rightarrow \overline{\mathbb{R}}$ two functions. The function $g$ is called $f$-quasiconvex in $x$ if for any finite subset $A$ of $X, g(x, y) \leq \max _{u \in A} f(u, y)$, for any $x \in \operatorname{co} A$ and all $y \in Y$.

From Theorem 4, we obtain an analytic alternative and a minimax inequality.

Theorem 7. Let $X$ be an almost convex admissible subset of a t.v.s. and $Y$ a nonempty convex subset in an l.c.s., both of them compact. Let $\alpha \leq \beta<\gamma$ be numbers and $f, g, s: X \times Y \rightarrow \overline{\mathbb{R}}$ three functions satisfying the following conditions:

(1) the sets $\{(x, y) \in X \times Y: f(x, y) \leq \alpha\}$ and $\{(x, y) \in X \times Y$ : $s(x, y) \geq \gamma\}$ are closed in $X \times Y$;

(2) $s(x, y) \leq g(x, y)$ for all $(x, y) \in X \times Y$;

(3) $g$ is $f$-quasiconvex in $y$;

(4) for each $y \in Y,\{x \in X: s(x, y) \geq \gamma\}$ is acyclic; 
(5) for each $x \in X$, the set $\{y \in Y: g(x, y) \leq \beta\}$ is closed in $Y$; Then at least one of the following assertions holds:

(a) There exists $x_{0} \in X$ such that $f\left(x_{0}, y\right)>\alpha$ for all $y \in Y$.

(b) There exists $y_{0} \in Y$ such that $s\left(x, y_{0}\right)<\gamma$ for all $x \in X$.

Proof. We define the maps $F, G, S: X \multimap Y$ by

$$
\begin{aligned}
& F(x)=\{y \in Y: f(x, y) \leq \alpha\}, \\
& G(x)=\{y \in Y: g(x, y) \leq \beta\}, \\
& S(x)=\{y \in Y: s(x, y)<\gamma\} .
\end{aligned}
$$

Since the graphs of $F$ and $S^{*}$ are closed and $X$ and $Y$ are compact, $F$ and $S^{*}$ are u.s.c. with compact values. Since $\beta<\gamma$ and $s(x, y) \leq g(x, y)$ for all $(x, y) \in X \times Y, G(x) \subset S(x)$ for all $x \in X$. By (5), $G$ has compact values. Let $x \in X$ and $y \in \operatorname{co} F(x)$. Then for some finite subset $A$ of $F(x), y \in \operatorname{co} A$. By $(3), g(x, y) \leq \max _{u \in A} f(x, u) \leq \alpha \leq \beta$, so $\operatorname{co} F(x) \subset G(x)$ for all $x \in X$.

Therefore the maps $F, G, S$ satisfy all the requirements of Theorem 4, so at least one of the following assertions holds:

There exists $x_{0} \in X$ such that $F\left(x_{0}\right)=\emptyset$, that is $f\left(x_{0}, y\right)>\alpha$ for all $y \in Y$.

There exists $y_{0} \in Y$ such that $y_{0} \in \bigcap_{x \in X} S(x)$, that is $s\left(x, y_{0}\right)<\gamma$ for all $x \in X$.

Theorem 8. Let $X$ be an almost convex admissible subset of a t.v.s. and $Y$ a nonempty convex subset in an l.c.s., both of them compact. Let $f, g, s: X \times Y \rightarrow \overline{\mathbb{R}}$ be three functions satisfying conditions (2), (3) in Theorem 7 and the following conditions:

$\left(1^{\prime}\right) f$ is l.s.c. and $s$ is u.s.c. on $X \times Y$;

$\left(4^{\prime}\right)$ for each $y \in Y$ and real number $\gamma,\{x \in X: s(x, y) \geq \gamma\}$ is acyclic;

$\left(5^{\prime}\right)$ for each $x \in X, g(x, y)$ is l.s.c. in $y$;

Then $\inf _{y \in Y} \max _{x \in X} s(x, y) \leq \sup _{x \in X} \min _{y \in Y} f(x, y)$.

Proof. First let us observe that if $f$ is l.s.c. on $X \times Y$, then for each $x \in X, f(x, \cdot)$ is l.s.c. in $y$ and therefore its minimum $\min _{y \in Y} f(x, y)$ on the compact set $Y$ exists. Similarly $\sup _{x \in X} s(x, y)$ can be replaced by $\max _{x \in X} s(x, y)$.

Suppose the conclusion would be false and choose real numbers $\alpha, \beta, \gamma$ such that

$$
\sup _{x \in X} \min _{y \in Y} f(x, y)<\alpha \leq \beta<\gamma<\inf _{y \in Y} \max _{x \in X} s(x, y) .
$$


Even the function $f, g, s$ satisfy all the requirements of Theorem 7, but

if (a) happens, then $\sup _{x \in X} \min _{y \in Y} f(x, y) \geq \min _{y \in Y} f\left(x_{0}, y\right)>\alpha$, a contradiction

if (b) happens, then $\inf _{y \in Y} \max _{x \in X} s(x, y) \leq \max _{x \in X} s\left(x, y_{0}\right)<\gamma$, a contradiction.

Acknowledgement. This paper is based on a part of the plenary talk given by the first author at the 2012 International Conference of the Honam Mathematical Society, held at Jeju National University, Jeju, Korea on June 15-17, 2012. He would like to express his sincere gratitude to the kind hospitality of the organizers of the conference.

\section{References}

[B] M. Balaj, Alternative principles and their applications, J. Glob. Optim. DOI 10.1007/s10898-010-9612-y.

[F] K. Fan, Some properties of convex sets reated to fixed point theorem, Math. Ann. 266 (1984), 519-537.

[L] M. Lassonde, Fixed points of Kakutani factorizable multifunctions, J. Math. Anal. Appl. 152 (1990), 46-60.

[M1] E. Michael, Continuous selections. I, Ann. Math. 63(2) (1956), 361-381.

[M2] E. Michael, A theorem on semi-continuous set-valued functions, Duke Math. J. 26 (1959), 647-651.

[P1] S. Park, Fixed point theory of multifunctions in topological vector spaces, II, J. Korean Math. Soc. 30 (1993), 413-431.

[P2] S. Park, Foundations of the KKM theory via coincidences of composites of upper semicontinuous maps, J. Korean Math. Soc. 31 (1994), 493-520.

[P3] S. Park, Coincidence theorems for the better admissible multimaps and their applications, Nonlinear Anal. 30 (1997), 4183-4191.

[P4] S. Park, A unified fixed point theory of multimaps on topological vector spaces, J. Korean Math. Soc. 35 (1998), 803-829. Corrections, ibid. 36 (1999), 829-832.

[P5] S. Park, Fixed points of multimaps in the better admissible class, J. Nonlinear Convex Anal. 5 (2004), 369-377.

[P6] S. Park, Fixed point theorems for better admissible multimaps on almost convex sets, J. Math. Anal. Appl. 329 (2007), 690-702.

[P7] S. Park, Applications of multimap classes in abstract convex spaces, J. Nat. Acad. Sci., ROK, Nat. Sci. Ser. 51(2) (2012), to appear.

Sehie Park

The National Academy of Sciences, Republic of Korea, Seoul 137-044; and 
Department of Mathematical Sciences, Seoul National University, Seoul 151-747, Korea.

E-mail: shpark@math.snu.ac.kr; parkcha38@daum.net

Hoonjoo Kim

Department of Mathematical Education, Sehan University, YoungAm-Gun, Chunnam, 530-702, Korea.

E-mail:hoonjoo@ sehan.ac.kr 